\title{
NeuroXNet: Creating A Novel Deep Learning Architecture that Diagnoses Neurological Disorders, Finds New Blood Biomarkers, and Assesses Surgical, Drugs, and Radiation Treatment Plans Using Medical Imaging and Genomic Data
} Vaibhav Mishra ${ }^{1}$

West Laurens High School, 3692 GA-257, Dexter, GA 31019

\begin{abstract}
Neurodegenerative diseases and cancerous brain tumors cause millions of patients worldwide to be fatally ill and face cognitive impairment each year. Current diagnosis and treatment of these neurological conditions take many days, are sometimes inaccurate, and use invasive approaches that could endanger the patient's life. Thus, this study's purpose is the creation of a novel deep learning model called NeuroXNet, which uses MRI images and genomic data to diagnose both neurodegenerative diseases like Alzheimer's disease, Parkinson's disease, and Mild Cognitive Impairment as well as cancerous brain tumors, including glioma, meningioma, and pituitary tumors. Moreover, the model helps find novel blood biomarkers of differentially expressed genes to aid in diagnosing the six neurological conditions. Furthermore, the model uses patient genomic data to give additional recommendations for treatment plans that include various treatment approaches, including surgical, radiation, and drugs for higher patient survival for each class of the disease. The NeuroXNet model achieves a training accuracy of $99.70 \%$, a validation accuracy of $100 \%$, and a test accuracy of $94.71 \%$ in multi-class classification of the six diseases and normal patients. Thus, NeuroXNet reduces the chances of misdiagnosis, helps give the best treatment options, and does so in a time/cost-efficient manner. Moreover, NeuroXNet efficiently diagnoses diseases and recommends treatment plans based on patient data using relatively few parameters causing it to be more cost and time-efficient in providing non-invasive approaches to diagnosis and treatment for neurological disorders than current procedures.
\end{abstract}

Keywords: Alzheimer's Disease; Parkinson's Disease; Mild Cognitive Impairment (MCI); Brain Tumors; Medical Imaging; Neuroscience; Blood Biomarkers; Genomics 


\section{Introduction}

\subsection{Background}

Neurological disorders continue to affect millions of people worldwide, with diseases leading to loss of cognitive function, a decline in memory, and even death. These diseases contribute to nearly a trillion dollars of healthcare spending and drastically change the lives of those affected. With the advent of new medical imaging and computational techniques, it has become possible to use large amounts of imaging data to build and train deep learning models that can diagnose many diseases with high accuracy rates using clinical tests and medical imaging tests like MRI. Some of the most common neurodegenerative disorders include Alzheimer's disease, Parkinson's disease, and Mild Cognitive Impairment. In the United States, approximately 6.2 million people are affected by AD [6], 1 million people are affected with PD [7], and 11 million people are affected with MCI [8].

Alzheimer's Disease (AD) is the most common neurodegenerative disease that starts with MCI like symptoms of memory loss and cognitive decline and later evolves to damage neurons responsible for carrying out essential functions affecting everyday life. Eventually, AD can result in the death of a patient. Some possible causes of AD include beta-amyloid plaques that form outside the neurons and tau tangles inside neurons, which have been observed inside several AD patients [6]. MRI images can diagnose AD earlier by locating critical parts of the brain that signal a higher possibility of AD. Some of the primary areas used to diagnose $\mathrm{AD}$ in the brain are the hippocampus and the parietal lobes. However, in patients with $\mathrm{AD}$, the MRI will clearly show a decrease in the size of the hippocampus signaling a higher probability of AD. The parietal lobe is also a signal that radiologists look for in diagnosing AD through MRIs. The parietal lobe is responsible for sensory information and visual processing in our bodies. A size reduction in a patient's parietal lobe also signals a chance of AD in a patient. [18] Therefore, MRIs must be adequately interpreted and used to give an early diagnosis of patients to prevent $\mathrm{AD}$ from progressing. Mild Cognitive Impairment (MCI) is a neurological condition with symptoms that show loss of certain cognitive functions like memory or the ability to speak. MCI can convert to AD or other types of dementia over a few years, remain stabilized as MCI, or revert to a normal functioning brain. The most common treatment of MCI is Aducanumab, a drug used to treat AD. [8] One major problem of diagnosis of MCI is that MCI can be similar in symptoms to normal cognitive decline due to old age in many people, which causes many patients to ignore the signs and leave MCI untreated. This is harmful as MCI can later develop into AD over time. Therefore, it is crucial to diagnose and treat $\mathrm{MCI}$ in its early stages to avoid more cognitive loss in patients. 
Parkinson's Disease (PD) is a neurological disease that primarily damages the motor neurons disturbing a patient's movement ability. PD usually results in patients having worse conditions over more extended periods. However, PD can be treated with certain medications like Carbidopa-levodopa [10]. MRIs can help detect specific abnormalities to brain structures that signal PD in PD patients. Particularly in $\mathrm{PD}$, there are significant changes seen in the right parietal cortex, the putamen, and brain structures responsible for motor actions like the mesencephalic locomotor region. The right parietal lobe is chiefly responsible for various functions of the body, including taste, touch, and the somatic sensory cortex within it. The putamen has the function of controlling movement, and as such, these areas are critical for PD diagnosis [19].

Glioma tumors are located in the central nervous system, and various glial cells are found throughout the peripheral nervous system, including astrocytes, oligodendrocytes, and microglia. Glioma tumors include glioblastoma multiforme, astrocytoma, and oligodendroglioma. Oligodendroglioma can occur in either the temporal or frontal lobes and cause seizures and motor inability. This study focuses on more lethal kinds of brain tumors, including anaplastic astrocytoma (which appears more commonly in the cerebral hemisphere) and glioblastoma multiforme (which starts in the cerebrum in astrocyte cells) [26]. Pituitary tumors primarily occur in the anterior portions of the pituitary gland. Pituitary tumors cause vertigo, fatigue, and problems with visual perception [26]. Pituitary tumors are generally treated through surgery using transsphenoidal resection [26]. Meningioma tumors occur on the meninges of the brain and can cause vision problems as well as seizures [26].

\subsection{Previous Literature}

Through the usefulness of large amounts of data and advancements in medical imaging research, it has become possible to diagnose and even treat patients with diseases in various fields of medicine. A large amount of medical imaging research using MRI data has been used to classify AD from normal patients. For instance, Al- Khuzaie et al. [4] developed a new model, AlzNet, which achieved an accuracy of 99.30\% in diagnosing AD from normal using 2D MRI slices. One of the highest accuracies was achieved in AD diagnosis using machine learning models. Another paper [11] trained a CNN model to classify $\mathrm{AD}$, normal, and MCI patients using 3D MRI with an accuracy of 52.3\%.

Medical imaging research has also been extended into PD classification from normal patients. Multiple models have been developed that incorporate a machine learning approach to classify PD from MRI imaging. A research team led by Long [1] developed a model in which accuracy of $86.96 \%$ was achieved in classifying and diagnosing PD patients from normal patients. In [56], computational tools identify novel blood biomarkers in PD patients using gene ontology analysis and protein interactions. 
However, relatively few machine learning models have performed multiclass diagnosis in neurological disorders. A multiclass approach to classifying multiple neurodegenerative diseases was studied in very few papers, notably in [14], where AD, PD, and CN were classified with an accuracy of 90\% for $\mathrm{AD}, 90 \%$ for control from ADNI, $89 \%$ for control from PPMI, and 90\% for PD using transfer learning on the VGG19 model which performed the best out of the ResNet 50, Inception Net, and VGG16 model which were also tested in the paper and in another article by Tong et al. [15], where a five-class model was proposed that achieved a $75.2 \%$ that classified AD and other dementia-like diseases using the RUSBoost algorithm. Even in these studies, the model was limited to classifying only neurodegenerative diseases in lesser than five classes. Therefore, this study aims to solve the problem of multiclass diagnosis and treatment of neurological disorders.

\subsection{Purpose of Study}

This study proposes a novel deep learning architecture, NeuroXNet, which performs multiclass diagnosis of $\mathrm{AD}, \mathrm{PD}, \mathrm{MCI}$, glioma, meningioma, pituitary, and normal patients. In addition, NeuroXNet generates recommendations for treatment based on disease classification from its convolutional neural network $(\mathrm{CNN})$ model combined with the patient's genomic and clinical data. These recommendations include treatment plans for surgery, radiation, or drug therapy. This is the first CNN model which creates a novel architecture to study multiclass diagnosis instead of relying on previously built models like ResNet50 or VGG16. Furthermore, this model discovers new blood biomarkers using genomic data to further aid in diagnosing neurological conditions and reducing the chance of misdiagnosis. These genes can also be further studied to find possible targets for treatment research. Moreover, this model is the first that combines diagnosis with treatment plans for neurological disorders. Therefore, this model has great potential to be used in neurological medicine and provide a low-cost, efficient, and quick solution to patients worldwide.

\section{Materials and Methods}

\subsection{Data Acquisition and Description for MRI Classifier}

Data of $\mathrm{AD}$ and MCI patients used in the paper was obtained from the Alzheimer's Disease Neuroimaging Initiative (ADNI), a free open source database (adni.loni.usc.edu), and data for the PD and normal patients was obtained from the Parkinson's Progression Markers Initiative (PPMI) database (ppmi-info.org). Data for MRI of patients with glioma, meningioma, and pituitary tumors were acquired from Kaggle's Brain Tumor Dataset [22]. For each of the seven AD, PD, MCI, Pituitary Tumor, Glioma Tumor, Meningioma Tumors, and Normal Patients, 1000 Brain MRI images were collected NIFTI format 
medRxiv preprint doi: https://doi.org/10.1101/2021.12.13.21267728; this version posted December 14, 2021. The copyright holder for this preprint (which was not certified by peer review) is the author/funder, who has granted medRxiv a license to display the preprint in perpetuity.

It is made available under a CC-BY-NC 4.0 International license .

from the respective databases for a total of 7000 images. All the MRIs were T1-weighted and equally distributed to reduce model bias. These images were then converted to png format using the MRIcro tool. The images were split into training/validation/test folders using a 70\%/30\%/10\% ratio. Table 1 shows the number of patient MRIs that were split into Training, Validation, and Test sets for each disease class.

\begin{tabular}{ccccc}
\hline & Table 1: Data Split Into Training, Validation, and Test Sets & & \\
\hline & Training Set & Validation Set & Test Set & Total \\
\hline Alzheimer's Disease & 700 & 300 & 100 & 1000 \\
\hline Parkinson's Disease & 700 & 300 & 100 & 1000 \\
\hline Mild Cognitive Impairment & 700 & 300 & 100 & 1000 \\
\hline Glioma Tumor & 700 & 300 & 100 & 1000 \\
\hline Meningioma Tumor & 700 & 300 & 100 & 1000 \\
\hline Pituitary Tumor & 700 & 300 & 100 & 1000 \\
\hline Normal Control & 700 & 300 & 100 & 1000 \\
\hline Total & 4900 & 2100 & 700 & 7000 \\
\hline
\end{tabular}

Table 1: Number of Patients per Data Set Folder

The demographic data including age and gender for the neurodegenerative classes is shown below in Table 2:

\begin{tabular}{cccccc}
\hline & \multicolumn{4}{c}{ Table 2: Demographic Data of Neurodegenerative Disease Classes } \\
\hline & Number of Subjects & Number of Male Subjects & Number of Female Subjects & Average Age & Range of Age \\
\hline Alzheimer's Disease & 1000 & 307 & 693 & $77.73 \pm 5.51$ & {$[72,87]$} \\
\hline Parkinson's Disease & 1000 & 617 & 383 & $60.39 \pm 8.43$ & {$[39,76]$} \\
\hline Mild Cognitive Impairment & 1000 & 462 & 338 & $75.74 \pm 8.98$ & {$[58,92]$} \\
\hline Normal Control & 1000 & 318 & 682 & $60.93 \pm 10.89$ & {$[31,81]$} \\
\hline Note: Average Age represents agetstandard deviation and range of age represents [min,max]. & &
\end{tabular}

Table 2: Demographic Data for Neurodegenerative Diseases

\subsection{Data Preprocessing for Diagnosis}

After being split into their respective folder, each MRI image was normalized by rescaling the size of each image. Then, data augmentation was applied, including shearing, zooming, and flipping the data to decrease overfitting and improve the model's overall accuracy. The data was preprocessed to reduce the amount of data bias the model gains and helps make the model regularize to fit all ranges of MRI images.

\subsection{Deep Learning Models Overview}

Convolutional Neural Networks (CNNs) are a class of deep neural networks part of supervised machine learning that are most widely used for visual image processing, including medical imaging. CNN's use multiple layers to take input images, find patterns within them, and learn from certain features 
within the pictures. They then use the learned weights and biases to output the image's class. A convolutional layer is the centerpiece of the NeuroXNet model, which uses a set of weights and biases (also called factors) that learns over time.

\subsection{Identification of Novel Biomarkers}

Identifying biomarkers from blood, tissue, or another type of body cell for early detection of neurological diseases was one of the main focuses of this study. Blood biomarkers are analyzed with microarrays to gather data on gene expression for thousands of genes and subsequently used as a noninvasive neurological test for diagnosis and research in finding new treatments. This study utilized genomic datasets from Gene Expression Omnibus. The data used in this study consisted of the series GSE74385 [46, 47] for meningioma tumor classification, GSE31095 [48] for glioma tumors, GSE4488 [49] for pituitary tumors, GSE63063 [50] for MCI classification, GSE6613 [51, 52] for PD classification, and GSE4226 [53,54] for AD classification. Each gene expression study was used to find differentially expressed genes that would serve as blood biomarkers for diagnosing the specific neurological disorder. The NeuroXNet model used to classify patients by genomic data.

Using GEO2R, the patients were split into two groups for each series number, with one group consisting of the specific disease class and the other group with normal patients. Then, all differentially expressed genes with a p-value of less than 0.05 were used to generate a protein interaction network using the STRING tool. The protein interaction network was then used to identify hub genes with a high number of nodes using the Cytoscape tool. The hub genes are the differentially expressed genes that are over-expressed in patients with certain neurological conditions and are potential biomarkers for the disease. These hub genes were sorted by degree (number of nodes connected to) and used for gene ontology analysis using the PANTHER tool, which gave the false positive rate, fold enrichment, and pvalues for the specific genes present in biological, molecular, and cellular processes.

The fold change represents the ratio of the average gene expression in the experimental group vs. the control group. This study focused on the over-expressed genes with fold change values greater than 1.

\subsection{NeuroXNet Model Description}

NeuroXNet is the novel CNN model created in this study that uses patient MRI data and genomic data to diagnose and give treatment recommendations to patients, including surgical, radiation, and drug treatments to improve patient survivability. First, the model inputs MRI images, preprocesses them and sends them to the NeuroXNet Model. The model uses pre-trained weights to classify the patient's MRI as $\mathrm{AD}, \mathrm{PD}, \mathrm{MCI}$, glioma tumor, meningioma tumor, pituitary tumor, or normal. Next, genomic data of the 
patient is entered in as a secondary input and used to diagnose the patient again to ensure an accurate diagnosis. The model diagnoses patients using its pre-trained algorithm from blood biomarkers which it found beforehand. The classification information is then passed through the treatment tool of the NeuroXNet model, which matches the classified disease to its best treatment plan taking into account the susceptibility of the disease to drug treatments, radiation treatments, or surgical treatments starting from non-invasive options first that provide the most minor side effects to the patient and eliminating options that have low rates of survival based on patient's genomic data. The process is shown in Figure 1 below:

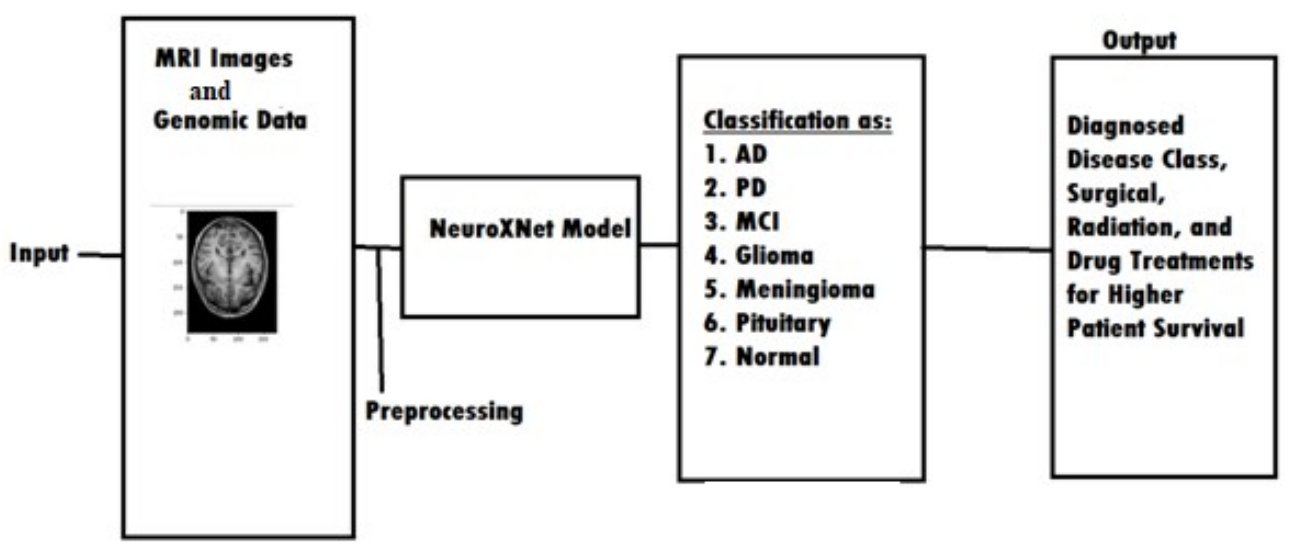

Figure 1: NeruoXNet Model Workflow Analysis

The next three sections detail the kind of algorithmic process that the NeuroXNet model goes through in suggesting treatment procedures for patients.

\subsection{Surgical Treatment Procedures}

For surgical procedures, NeuroXNet focuses on only widely used methods and can cause significant improvements to patient health. The model makes recommendations based on the diagnosed disease input from the result of its $\mathrm{CNN}$ model.

For patients diagnosed with PD, the model considers only the two main surgical methods used by surgeons: Deep Brain Stimulation (DBS) and Duopa. DBS is a surgical procedure that involves placing electrodes into the brain with an impulse generator battery that is connected to a wire that passes through the head and shoulder. DBS delivers electrical stimulation to the subthalamic nucleus and the globus pallidus internus, which control movements that cause tremors to reduce PD symptoms [28]. DBS is used for patients that are in the early stages of PD and experience uncontrolled tremors [28]. 
For patients that are diagnosed with MCI, the NeuroXNet model consists of mainly one surgical treatment procedure: Transcutaneous Electrical Nerval Stimulation (TENS). TENS is a medical procedure that involves applying an electrical current to electrons located on the skin in order to reduce pain in patients. TENS improves cognitive functioning, as well as counters, sleep disorders, and neural degeneration [30].

For patients that have pituitary tumors, the model either suggests a craniotomy or an endoscopic transnasal transsphenoidal approach [31]. A craniotomy is performed by making an incision on the scalp, after which a portion of the skull is removed. Then, as much of the tumor that can be removed without harming healthy brain tissue is taken out. Next, the skull part is replaced, and the incision is closed [32]. An endoscopic transnasal transsphenoidal approach involves removing the tumor through the nasal pathway [31]. The model suggests craniotomy only if the tumor is greater than or equal to $10 \mathrm{~mm}$ in diameter size. Otherwise, it offers an endoscopic transnasal transsphenoidal approach.

For diagnosis as a meningioma tumor, if the tumor is found in the ventricular chambers, neuroendoscopic surgery is recommended by the model. Otherwise, if the tumor is large (10mm or more in diameter size), a craniotomy is suggested. If the tumor is small, then an endoscopic endonasal approach is recommended. Intraventricular infusion involves delivering antibodies into the brain to provide immunization against amyloid-beta peptides in the amyloid plaques that are found in AD patients [33]. Gene therapy consists in injecting brain-derived neurotrophic factors (BDNF) into the CNS to increase neuronal growth to reverse AD progression [34]. Cerebrospinal fluid shunting involves draining CSF from ventricles of the brain. This causes fresh CSF to form, which are free from tau proteins (a major cause of $\mathrm{AD}$ ). CSF shunting is seen to decrease tau proteins by $60 \%$ in $\mathrm{AD}$ patient brains [35]. 


\begin{tabular}{|c|c|c|}
\hline & \multicolumn{2}{|c|}{ Table 5: Surgical Treatment Summary for NeuroXNet } \\
\hline & Determining Factor & Result \\
\hline \multirow{2}{*}{ PD } & Early Stages of PD & Deep Brain Stimulation \\
\hline & Late Stages of PD & Duopa \\
\hline \multirow{2}{*}{$\mathrm{MCl}$} & Patient Needs Pain Control & $\begin{array}{l}\text { Transcutaneous Electrical Nerval } \\
\text { Stimulation (TENS) } \\
\end{array}$ \\
\hline & Does Not Need Pain Control & Use Medicine or Alternative Approaches \\
\hline \multirow{2}{*}{ Pituitary } & $\begin{array}{l}\text { Tumor grater than or equal to } 10 \mathrm{~mm} \text { in diameter } \\
\text { size }\end{array}$ & Craniotomy \\
\hline & Tumor less than $10 \mathrm{~mm}$ in diameter size & $\begin{array}{l}\text { Endoscopic Transnasal Transsphenoidal } \\
\text { Approach }\end{array}$ \\
\hline \multirow{3}{*}{ Meningioma } & Tumor found in ventricular chambers & Neuroendoscopic Surgery \\
\hline & $\begin{array}{l}\text { Tumor grater than or equal to } 10 \mathrm{~mm} \text { in diameter } \\
\text { size }\end{array}$ & Craniotomy \\
\hline & Tumor less than $10 \mathrm{~mm}$ in diameter size & Endoscopic Endonasal \\
\hline \multirow{4}{*}{ Glioma } & Tumor has caused build up of CSF & Shunt Placement \\
\hline & Surgery Needed to Test Tumor Removal Effects & Brain Mapping and Awake Surgery \\
\hline & Surgery Needed to Test Tumor Tissue & Surgical Biopsy \\
\hline & $\begin{array}{l}\text { Surgery Required to Remove Tumor Without Any } \\
\text { Functionality Tests }\end{array}$ & Surgical Resection \\
\hline \multirow{4}{*}{$A D$} & Patient Needs Pain Control & $\begin{array}{l}\text { Transcutaneous Electrical Nerval } \\
\text { Stimulation (TENS) }\end{array}$ \\
\hline & Patient in Early Stages of AD & Cerebrospinal Fluid Shunting \\
\hline & Patient in Moderate Stage of AD & Gene Therapy \\
\hline & Patient in Late Stage of AD & Intraventricular Infusion \\
\hline
\end{tabular}

\subsection{Radiation Treatment Procedures}

Patients classified as having AD have been recorded to see vast improvements when exposed to low doses of ionizing radiation [42]. Advances in cognitive and motor functions were observed on AD patients exposed to the amounts of radiation [42]. Thus, NeuroXNet proposes low doses of ionizing radiation for patients who seek a non-invasive approach.

NeuroXNet recommends patients with meningioma tumors that have tumors near crucial brain structures IMRT. Otherwise, for large tumors, fractionated stereotactic radiotherapy is used. If the meningioma cannot be removed through the previous methods, then proton beam radiation is recommended to be used [44].

For patients with glioma tumors, there are two main types of radiation therapy that NeuroXNet recommends: IMRT and Image-Guided Radiation Therapy (IGRT). IGRT uses imaging software during treatment to adjust positions as needed. IMRT uses software to target radiation on the tumor and eliminate it directly. For tumors that are glioblastomic in nature, radiation therapy can be combined with the intake of temozolomide. Glioblastoma can also be treated by proton therapy [45]. NeuroXNet generates a treatment plan based on if the tumor is glioblastoma and if the patient's tumor would be removed better through real-time imaging or not. 


\begin{tabular}{|l|l|l|}
\hline \multicolumn{3}{|c|}{ Table 6: Radiation Treatment Summary for NeuroXNet } \\
\hline & Determining Factor & Result \\
\hline PD & Patient Needs Non-Invasive Approach & Stereotactic Radiosurgery \\
\hline MCI & No Radiation Treatment Availaible & No Radiation Treatment Availaible \\
\hline \multirow{3}{*}{ Pituitary } & Brain Tumor is Near Sensitive Areas & Intensity-Modulated Radiation Therapy \\
\cline { 2 - 4 } & Brain Tumor Outside Sensitive Areas & $\begin{array}{l}\text { Proton Beam Radiation or Stereotactic } \\
\text { Radiotherapy }\end{array}$ \\
\hline \multirow{2}{*}{ Meningioma } & Tumor near Crucial Brain Structures & Stereotactic Radiotherapy \\
\cline { 2 - 4 } & Tumor Outside Crucial Brain Structures & Proton Beam Radiation \\
\hline \multirow{3}{*}{ Glioma } & Tumor does not need real-time imaging & Intensity-Modulated Radiation Therapy \\
\cline { 2 - 4 } & Tumor needs real-time imaging & Image-Guided Radiation Therapy \\
\cline { 2 - 3 } & Tumor is glioblastoma & Proton Radiation Therapy + Temozolomide \\
\hline \multirow{2}{*}{ AD } & $\begin{array}{l}\text { Patient Needs Improvement in Cognitive and Motor } \\
\text { Functions }\end{array}$ & Low Doses of Ionizing Radiation \\
\hline
\end{tabular}

\subsection{Drug Treatment Procedures}

For AD patients, one of the prime medical drugs used for treatment is Aducanumab which attacks beta-amyloid proteins to reduce amyloid plaque sizes, improving cognitive function and other AD-related symptoms [38]. Other drugs that may be used include cholinesterase inhibitors like Donepezil or Rivastigmine, which improves memory and thinking functions in the brain; glutamate regulators like memantine which will enhance overall cognitive functions; and orexin receptor antagonists with suvorexant to improve behavioral symptoms [38].

The most effective drug treatment plan for patients diagnosed with PD is using levodopa with carbidopa. Levodopa is a CNS agent converted to dopamine in the brain, helping ease PD symptoms as a lack of dopamine causes PD. Carbidopa is a decarboxylase inhibitor used with levodopa and stops levodopa from being converted into dopamine before it crosses the blood-brain barrier [36]. However, this combination of drugs does not work for some patients and causes dyskinesia. This is seen to be because of DNA methylation that changes the structure of the striatal methylome or causes methyl groups to be lost, causing Levodopa-induced dyskinesia [37].

The best option for meningioma tumors is to get surgical treatment to remove their tumors. However, drug treatment can also be used with limited efficiency for those unable to get surgical treatment. The most common drugs used to treat meningioma tumors include antibody proteins like Interferon, Bevacizumab, and Hydroxyurea which prevent the growth of cancerous cells and decrease blood flow to the tumor by providing support to the immune system [39].

One of the most effective drug treatments for glioma tumors is Temozolomide (TMZ), which reduces tumor size by fastening the DNA of the cancerous cells and preventing cell division. TMZ is 
medRxiv preprint doi: https://doi.org/10.1101/2021.12.13.21267728; this version posted December 14, 2021. The copyright holder for this preprint (which was not certified by peer review) is the author/funder, who has granted medRxiv a license to display the preprint in perpetuity.

It is made available under a CC-BY-NC 4.0 International license .

more effective than other drugs because it can pass through the blood-brain barrier and directly attack the cancerous tumor inside [23]. However, for patients with the O6-methylguanine methyltransferase (MGMT) protein over-expressed in glioma cells, TMZ could result in glioma cells becoming resistant and not being treated through medicine alone. This occurs because MGMT encodes a DNA repair enzyme which can cause the glioma cells to resist treatment with TMZ. TMZ treats both glioblastoma multiforme and anaplastic astrocytoma and is widely used with inhibitors of MGMT enzyme to counter any resistance that the cancerous cells build to the drug [24]. One of the most effective combinations of medications includes TMZ combined with MGMT-modified $\gamma \delta$ T cells, which effectively prevents tumor growth and immunizes tumors through NKG2D ligands [25].

\begin{tabular}{|c|c|c|}
\hline & Determining Factor & Result \\
\hline \multirow{2}{*}{ PD } & $\begin{array}{l}\text { Patient Does Not Have DNA Methylation Occuring In Brain } \\
\text { Cells }\end{array}$ & Levodopa+Carbidopa \\
\hline & Patient Has DNA Methylation Occuring In Brain Cells & $\begin{array}{l}\text { Levodopa+Carbidopa+Drugs used to prevent } \\
\text { Levodopa-Induced Dyskenesia }\end{array}$ \\
\hline $\mathrm{MCl}$ & No Medication Treatment Available & No Medication Treatment Available \\
\hline \multirow{3}{*}{ Pituitary } & Pituitary tumor secretes excess cortisol & Ketoconazole \\
\hline & Pituitary tumor secretes excess growth hormone & Pegvisomant \\
\hline & Pituitary tumor secretes excess prolactin & Bromocriptine+Cabergoline \\
\hline \multirow{2}{*}{ Meningioma } & Patient be subjected to surgery & Use surgical options \\
\hline & Patient cannot be subjected to surgery & Interferon or Bevacizumab or Hydroxyurea \\
\hline \multirow[t]{2}{*}{ Glioma } & Patient has over expression of MGMT gene & $\begin{array}{l}\text { Temozolomide+Radiation or } \\
\text { Temozolomide+MGMT-gamma-delta modified } \\
\text { Tcells }\end{array}$ \\
\hline & Patient does not have over expression of MGMT gene & Temozolomide \\
\hline \multirow{4}{*}{ AD } & Patient needs overall improvement in all AD symptoms & Aducanumab \\
\hline & $\begin{array}{l}\text { Patient needs overall improvements only in memory and } \\
\text { thinking functions }\end{array}$ & Donepezil or Rivastigmine \\
\hline & Patient needs overall improvements in cognitive functions & Memantine \\
\hline & Patient needs overall improvement in behavioral functions & Orexin receptor antagonists with suvorexant \\
\hline
\end{tabular}

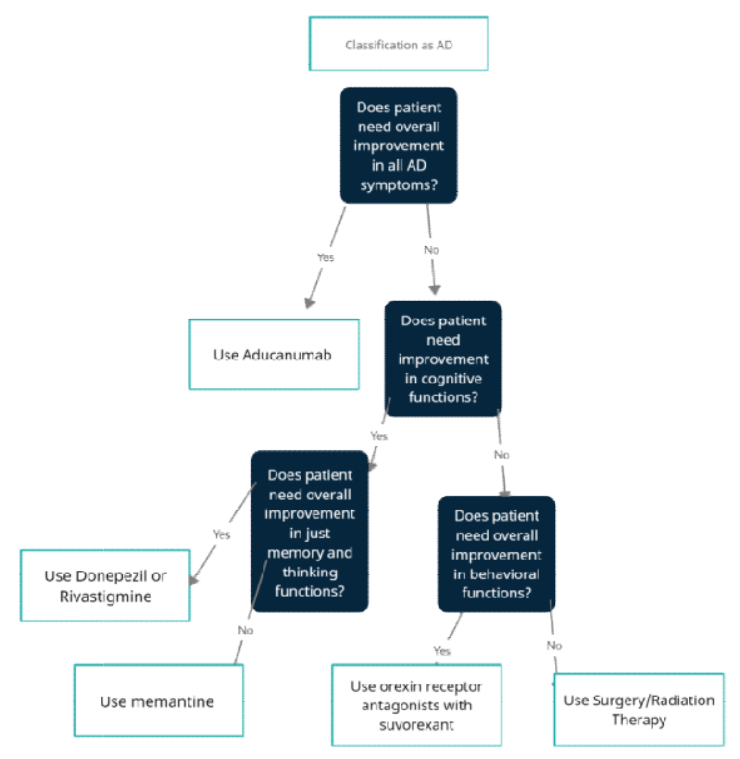

Figure 2: Example of NeuroXNet Algorithm for AD Drug Treatment 


\section{Results and Analysis}

\subsection{Results for Identification of Blood Biomarkers}

\begin{tabular}{|c|c|c|}
\hline $\begin{array}{c}\text { Hub } \\
\text { Gene }\end{array}$ & Degree & Clustering Coefficient \\
\hline CENPF & 7 & 1 \\
\hline DLGAP5 & 7 & 0.933333 \\
\hline $\begin{array}{c}\text { KIAA010 } \\
1\end{array}$ & 7 & 0.714286 \\
\hline TOP2A & 7 & 0.714286 \\
\hline BUB1B & 7 & 0.666667 \\
\hline
\end{tabular}

\section{Figure 3: Hub Genes for PD}

\begin{tabular}{|c|c|c|c|c|c|}
\hline GO Biological Process & Observed Gene Count & Expected Gene Count & Fold Enrichment & P-value & False Discovery Rate \\
\hline $\begin{array}{c}\text { neuron-glial cell } \\
\text { signaling (GO:0150099) }\end{array}$ & 3 & 0.05 & 58.29 & $5.62 \mathrm{E}-05$ & $2.10 \mathrm{E}-02$ \\
\hline $\begin{array}{l}\text { positive regulation of } \\
\text { heart rate by } \\
\text { epinephrine- } \\
\text { norepinephrine } \\
\text { (GO:0001996) }\end{array}$ & 3 & 0.06 & 48.57 & 8.37E-05 & 2.63E-02 \\
\hline $\begin{array}{l}\text { positive regulation of } \\
\text { blood pressure by } \\
\text { epinephrine- } \\
\text { norepinephrine } \\
\text { (GO:0003321) }\end{array}$ & 3 & 0.07 & 41.63 & 1.19E-04 & $2.96 \mathrm{E}-02$ \\
\hline $\begin{array}{c}\text { regulation of systemic } \\
\text { arterial blood pressure } \\
\text { by norepinephrine- } \\
\text { epinephrine } \\
\text { (GO:0001993) }\end{array}$ & 3 & 0.09 & 32.38 & $2.14 \mathrm{E}-04$ & 4.11E-02 \\
\hline GO Molecular Function & Observed Gene Count & Expected Gene Count & Fold Enrichment & P-value & False Discovery Rate \\
\hline $\begin{array}{c}\text { alpha1-adrenergic } \\
\text { receptor activity } \\
\text { (GO:0004937) }\end{array}$ & 3 & 0.03 & 97.15 & 2.04E-05 & $1.99 \mathrm{E}-02$ \\
\hline $\begin{array}{c}\text { protein domain specific } \\
\text { binding (GO:0019904) }\end{array}$ & 21 & 7.22 & 2.91 & $1.66 \mathrm{E}-05$ & $2.02 \mathrm{E}-02$ \\
\hline GO cellular component & Observed Gene Count & Expected Gene Count & Fold Enrichment & P-value & False Discovery Rate \\
\hline $\begin{array}{c}\text { plasma membrane raft } \\
\text { (GO:0044853) }\end{array}$ & 9 & 1.21 & 7.41 & $6.26 \mathrm{E}-06$ & $1.24 \mathrm{E}-02$ \\
\hline $\begin{array}{c}\text { membrane } \\
\text { microdomain } \\
\text { (GO:0098857) }\end{array}$ & 14 & 3.5 & 4 & $1.74 \mathrm{E}-05$ & $1.73 \mathrm{E}-02$ \\
\hline $\begin{array}{l}\text { membrane raft } \\
\text { (GO:0045121) }\end{array}$ & 14 & 3.5 & 4 & $1.74 \mathrm{E}-05$ & $1.15 \mathrm{E}-02$ \\
\hline
\end{tabular}

Figure 4: Biological, Molecular, and Cellular Functions from PD Over expressed Gene

Five hub genes were found for PD patients with a degree of 7. These genes were CENPF, DLGAP5, KLAA0101, TOP2A, and BUB1B. CENPF or Centromere Protein F is related to the centromerekinetochore complex and is responsible for chromosome separation during mitosis. The gene is also 
differentially expressed in cancer patients. DLG Associated Protein 5 codes for protein and is also found in cancerous patients. DNA Topoisomerase II Alpha (TOP2A) is crucial in the transcription process, helping create enzymes for chromosome segregation and condensation. This gene also counters drug resistance in patients with ataxia-telangiectasia [55].

For the biological function of neuron-glial cell signaling, a fold enrichment value of 58.29 was observed in 3 over-expressed PD genes. In molecular functions, the alpha1-adrenergic receptor activity process had three genes with a fold enrichment of 97.15. This shows that the over-expressed genes are essential biomarkers of PD in patients, and the extremely low false discovery rate supports that this observance is not by random chance.

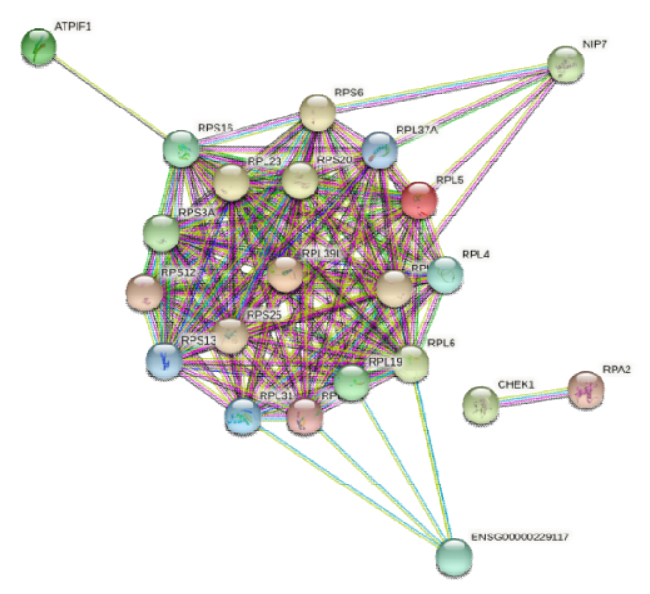

Figure 5: Protein Interaction Network of Biomarkers for AD

For AD blood biomarkers, nine hub genes were over-expressed in the patients with the condition. RPL24 had a degree of 18 , and the other eight genes each had a degree of 8 , showing that these genes interacted closely and were signals of AD in patients. Ribosomal Protein L24 (RPL24) codes for protein synthesis and is part of the ribosomal proteins family of L24E. The RPL series and RPS series of genes which are the hub genes, are associated with certain cancers. The model shows that the ubiquitin ligase inhibitor activity and ubiquitin-protein transferase inhibitor activity have extremely large fold enrichments for molecular processes. These hub genes are efficient signals for AD diagnosis and can be studied for possible treatment of the disease. 
medRxiv preprint doi: https://doi.org/10.1101/2021.12.13.21267728; this version posted December 14, 2021. The copyright holder for this preprint (which was not certified by peer review) is the author/funder, who has granted medRxiv a license to display the preprint in perpetuity.

It is made available under a CC-BY-NC 4.0 International license .

\begin{tabular}{|c|c|c|}
\hline Gene & Degree & Clustering Coefficient \\
\hline RPL24 & 18 & 0.830065359 \\
\hline RPL31 & 17 & 0.911764706 \\
\hline RPL19 & 17 & 0.911764706 \\
\hline RPL5 & 17 & 0.904411765 \\
\hline RPS16 & 17 & 0.882352941 \\
\hline RPL6 & 17 & 0.911764706 \\
\hline RPS6 & 17 & 0.904411765 \\
\hline RPL36A & 17 & 0.911764706 \\
\hline RPL37A & 17 & 0.904411765 \\
\hline
\end{tabular}

Figure 6: Hub Genes for AD

\begin{tabular}{|c|c|c|c|c|c|}
\hline GO Biological Process & Observed Gene Count & Expected Gene Count & Fold Enrichment & P-value & False Discovery Rate \\
\hline $\begin{array}{c}\text { negative regulation of } \\
\text { ubiquitin protein ligase } \\
\text { activity (GO:1904667) }\end{array}$ & 3 & 0.04 & 80.55 & $1.21 \mathrm{E}-05$ & $7.03 E-03$ \\
\hline $\begin{array}{l}\text { regulation of ubiquitin } \\
\text { protein ligase activity } \\
\text { (GO:1904666) }\end{array}$ & 4 & 0.07 & 55.85 & $1.35 \mathrm{E}-06$ & $8.17 \mathrm{E}-04$ \\
\hline $\begin{array}{l}\text { negative regulation of } \\
\text { ubiquitin-protein } \\
\text { transferase activity } \\
\text { (GO:0051444) }\end{array}$ & 3 & 0.06 & 52.36 & $3.76 \mathrm{E}-05$ & $1.85 \mathrm{E}-02$ \\
\hline $\begin{array}{c}\text { cytoplasmic translation } \\
\text { (GO:0002181) } \\
\end{array}$ & 17 & 0.35 & 48.64 & $9.49 E-24$ & $1.49 E-19$ \\
\hline $\begin{array}{l}\text { ribosomal large subunit } \\
\text { assembly (GO:0000027) }\end{array}$ & 3 & 0.08 & 38.79 & $8.51 E-05$ & $3.82 \mathrm{E}-02$ \\
\hline $\begin{array}{l}\text { GO Molecular } \\
\text { Component }\end{array}$ & Observed Gene Count & Expected Gene Count & Fold Enrichment & P-value & False Discovery Rate \\
\hline $\begin{array}{l}\text { ubiquitin ligase inhibitor } \\
\text { activity (GO:1990948) }\end{array}$ & 3 & 0.03 & $>100$ & $4.78 \mathrm{E}-06$ & $3.89 \mathrm{E}-03$ \\
\hline $\begin{array}{l}\text { ubiquitin-protein } \\
\text { transferase inhibitor } \\
\text { activity (GO:0055105) }\end{array}$ & 3 & 0.03 & $>100$ & $6.20 \mathrm{E}-06$ & $4.33 \mathrm{E}-03$ \\
\hline $\begin{array}{l}\text { ubiquitin-protein } \\
\text { transferase regulator }\end{array}$ & 4 & 0.07 & 60.71 & $1.00 \mathrm{E}-06$ & $1.22 \mathrm{E}-03$ \\
\hline $\begin{array}{l}\text { mRNA 5'-UTR binding } \\
\text { (GO:0048027) }\end{array}$ & 4 & 0.07 & 53.7 & $1.56 \mathrm{E}-06$ & $1.52 \mathrm{E}-03$ \\
\hline GO Cellular Component & Observed Gene Count & Expected Gene Count & Fold Enrichment & P-value & False Discovery Rate \\
\hline $\begin{array}{c}\text { cytosolic large ribosomal } \\
\text { subunit (GO:0022625) }\end{array}$ & 11 & 0.17 & 66.2 & $6.19 \mathrm{E}-17$ & 3.07E-14 \\
\hline $\begin{array}{l}\text { polysomal ribosome } \\
\text { (GO:0042788) }\end{array}$ & 6 & 0.09 & 63.47 & $1.27 E-09$ & $3.15 \mathrm{E}-07$ \\
\hline $\begin{array}{l}\text { cytosolic ribosome } \\
\text { (GO:0022626) }\end{array}$ & 18 & 0.3 & 61 & $9.38 E-27$ & $1.86 \mathrm{E}-23$ \\
\hline
\end{tabular}

Figure 7: Biological, Molecular, and Cellular Functions from AD Over expressed Genes

\begin{tabular}{|c|c|c|}
\hline Gene & Degree & Clustering Coefficient \\
\hline FYN & 7 & 0.142857143 \\
\hline SNRNP70 & 5 & 0.1 \\
\hline CHD4 & 5 & 0 \\
\hline
\end{tabular}

Figure 8: Hub Genes for MCI 
For MCI patients, three hub genes were found, namely: FYN, SNRNP70, and CHD4. FYN is responsible for cell growth control in the tyrosine kinase protein family. SNRNP70 is responsible for many types of diseases involving body tissue. These hub genes are essential in classifying MCI through blood samples and can serve as a way for neurologists to differentiate between MCI and AD patients because of the different hub genes for each disease.

\begin{tabular}{|c|c|c|}
\hline Gene & Degree & Clustering Coefficient \\
\hline PRKCA & 5 & 0 \\
\hline CYP7A1 & 4 & 0 \\
\hline FZD9 & 4 & 0.5 \\
\hline WNT5A & 4 & 0.5 \\
\hline LRP6 & 4 & 0.5 \\
\hline
\end{tabular}

\section{Figure 9: Hub Genes for Glioma Tumors}

Five hub genes were found for patients with glioma tumors, and two biological processes of calcium ion export and regulation of postsynaptic cytosolic calcium ion concentration had high enrichment values of 38.5 and 35, respectively. PRKCA is associated with other types of cancer.

\begin{tabular}{|c|c|c|}
\hline Gene & Degree & Clustering Coefficient \\
\hline BICC1 & 8 & 0.214285714 \\
\hline MPDZ & 7 & 0.142857143 \\
\hline COL4A2 & 7 & 0.333333333 \\
\hline GLI2 & 7 & 0.285714286 \\
\hline BTRC & 6 & 0.066666667 \\
\hline PLA2G15 & 5 & 0 \\
\hline CTLA4 & 5 & 0.1 \\
\hline
\end{tabular}

\section{Figure 10: Pituitary Hub Genes}

For patients with pituitary tumors, seven hub genes were found by the model. MPDZ proteins are responsible for HTR2C genes clot in the cell. Together, these hub genes can be used as non-invasive approaches for helping identify pituitary tumors in patients. 


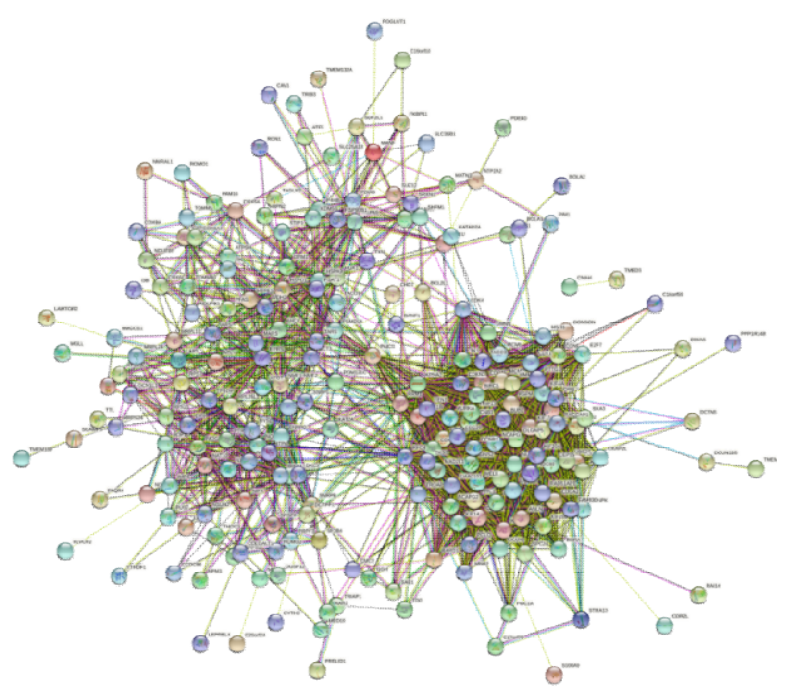

Figure 11: Protein Interaction Network for Meningioma Over Expressed Genes

One of the most significant results of the NeuroXNet model was the biomarkers it found for meningioma tumors. The model found 37 hub genes that were overly expressed in patients with meningioma tumors, and all had degrees greater than 64 . The model shows that the biological process of DNA replication preinitiation complex assembly has a high enrichment value of 78.31 among the associated hub genes. The cellular function of the cyclin B1-CDK1 complex also has a high enrichment value of 78.31. Some of the hub genes with the highest interactions included CDK1, CCNA2, AURKA, and BUB1 (also associated with PD patients). Cyclin-Dependent Kinase 1 (CDK1) has been associated with breast cancer and is involved in M-phase promoting factors. Cyclin A2 (CCNA2) is also in the same family of genes and is responsible for protein transition. The gene is also found in patients with other types of cancer.

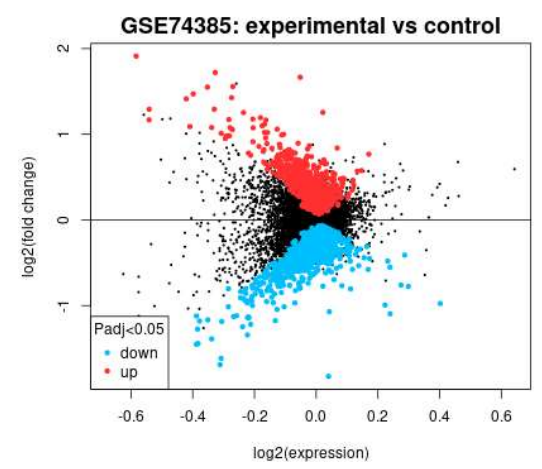

Figure 12: Mean Difference Plot for Meningioma Related Genes 


\begin{tabular}{|c|c|c|}
\hline Gene & Degree & Clustering Coefficient \\
\hline CDK1 & 82 & 0.648298705 \\
\hline CCNA2 & 76 & 0.746315789 \\
\hline AURKA & 76 & 0.723157895 \\
\hline BUB1 & 76 & 0.75122807 \\
\hline CDC20 & 75 & 0.76 \\
\hline KIF11 & 75 & 0.764324324 \\
\hline CDC45 & 74 & 0.759718623 \\
\hline CCNB1 & 74 & 0.763791188 \\
\hline ASPM & 74 & 0.778600518 \\
\hline NCAPG & 73 & 0.79718417 \\
\hline CCNB2 & 72 & 0.809859155 \\
\hline BIRC5 & 72 & 0.780125196 \\
\hline MAD2L1 & 72 & 0.781690141 \\
\hline DLGAP5 & 72 & 0.8157277 \\
\hline RFC4 & 72 & 0.631846635 \\
\hline UBE2C & 71 & 0.805231388 \\
\hline CENPF & 71 & 0.828973843 \\
\hline TPX2 & 71 & 0.824144869 \\
\hline MELK & 71 & 0.803219316 \\
\hline PTTG1 & 71 & 0.820925553 \\
\hline RACGAP1 & 70 & 0.805383023 \\
\hline PRC1 & 70 & 0.827329193 \\
\hline MCM2 & 70 & 0.776811594 \\
\hline KIF20A & 70 & 0.826915114 \\
\hline NDC80 & 69 & 0.825234442 \\
\hline NUSAP1 & 69 & 0.859335038 \\
\hline HMMR & 69 & 0.830349531 \\
\hline DTL & 69 & 0.794970162 \\
\hline CEP55 & 68 & 0.852941176 \\
\hline RAD51AP1 & 67 & 0.857078245 \\
\hline TRIP13 & 66 & 0.846153846 \\
\hline CDKN3 & 66 & 0.81025641 \\
\hline KIF4A & 66 & 0.854079254 \\
\hline CKS2 & 66 & 0.831235431 \\
\hline CENPA & 66 & 0.830769231 \\
\hline MCM6 & 65 & 0.808173077 \\
\hline FEN1 & 65 & 0.725480769 \\
\hline
\end{tabular}

Figure 13: Meningioma Hub Genes

\subsection{NeuroXNet Classification Results}

For my sequential model, NeuroXNet, the model starts with a convolutional layer with 32 filters, a stride of 2 , the same padding, and a kernel size of 3 . The layer takes in the input mages using an activation ReLU function. Next, the images are passed into a max-pooling layer with a pool size of 2 and a stride of 2 . Then, the images pass through another convolutional layer with filter size 128 . Next, the images pass into another max-pooling layer followed by a convolutional layer with filter size 128. Next, the images are passed into a batch normalization layer with a momentum of 0.8. Finally, the images are flattened through a flatten layer and are passed into the dense layer, which uses the softmax function to classify and diagnose the MRI images.

NeuroXNet was created using Keras with a Tensorflow backend and had eight layers. The model has 229,895 total parameters, which is relatively less than previously used models for disease diagnosis. The model architecture is shown below: 
medRxiv preprint doi: https://doi.org/10.1101/2021.12.13.21267728; this version posted December 14, 2021. The copyright holder for this preprint (which was not certified by peer review) is the author/funder, who has granted medRxiv a license to display the preprint in perpetuity.

It is made available under a CC-BY-NC 4.0 International license .

\begin{tabular}{|c|c|c|}
\hline $\begin{array}{l}\text { Layer (type) } \\
===========================\end{array}$ & $\begin{array}{l}\text { Output Shape } \\
=====================\end{array}$ & $\begin{array}{l}\text { Param \# } \\
===========\end{array}$ \\
\hline conv2d (Conv2D) & (None, 112, 112, 32) & 896 \\
\hline max_pooling2d (MaxPooling2D) & (None, 56, 56, 32) & $\theta$ \\
\hline conv2d_1 (Conv2D) & (None, $28,28,128)$ & 36992 \\
\hline max_pooling2d_1 (MaxPooling2 & (None, $14,14,128)$ & $\theta$ \\
\hline conv2d_2 (Conv2D) & (None, 7, 7, 128) & 147584 \\
\hline batch_normalization (BatchNo & (None, 7, 7, 128) & 512 \\
\hline flatten (Flatten) & (None, 6272) & $\theta$ \\
\hline 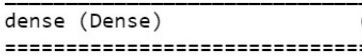 & 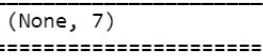 & $\begin{array}{l}43911 \\
==========\end{array}$ \\
\hline $\begin{array}{l}\text { Total params: } 229,895 \\
\text { Trainable params: } 229,639 \\
\text { Non-trainable params: } 256\end{array}$ & & \\
\hline
\end{tabular}

\section{Figure 14: NeuroXNet Model Architecture for Disease Diagnosis}

The model had an input of 6300 images from the Training and Validation folders, which is used to train itself. The model was run for 30 epochs and had a training accuracy of $99.79 \%$ with a loss of 0.0067 and a validation accuracy of $100 \%$ with a 0.0010 loss at the end of the 30 epochs. The training and validation accuracy and loss graphs over the epochs are shown below:

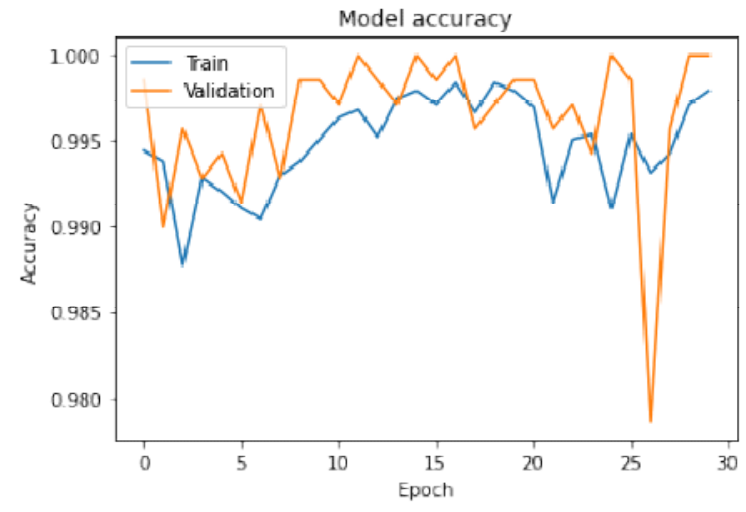

Figure 15: Training and Validation Model Accuracy 


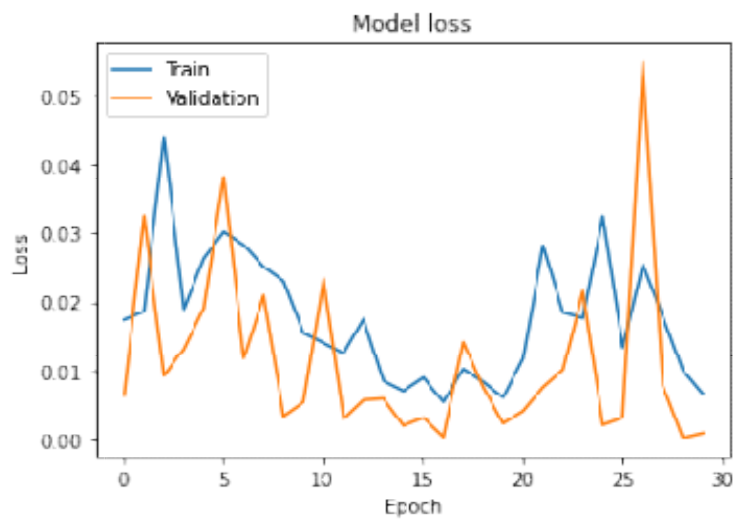

Figure 16: Training and Validation Model Loss

Then, the model ran the images on the test set comprising 700 images evenly split into seven classes. The confusion matrix for the testing data is shown below:

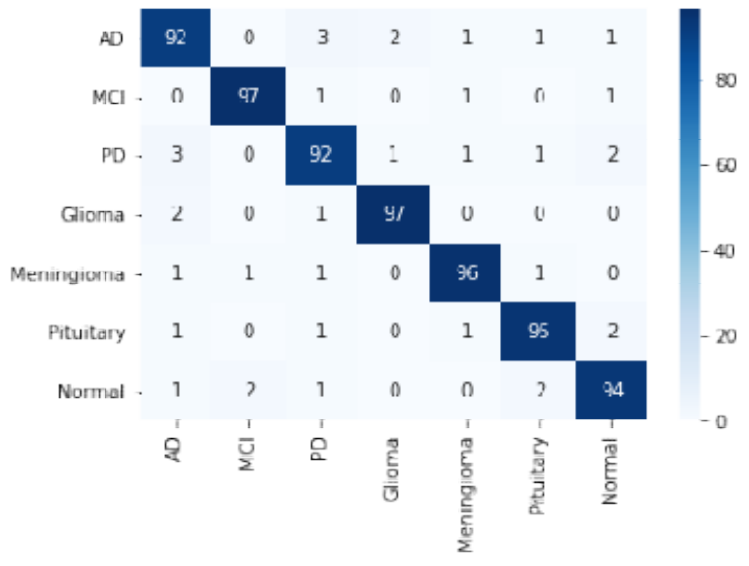

\section{Figure 17: Model Confusion Matrix and Heatmap}

The confusion matrix shows the number of images in each class and their respective predicted values compared to the actual values from the test set. The confusion matrix also helps visualize the model's performance on the test set through the heatmap colors. The diagonal values of 92, 97, 92, 97, 96, 95, and 94 represent the number of images that NeuroXNet correctly classified in each of the seven classes of AD, MCI, PD, Glioma, Meningioma, Pituitary, and Normal respectively. Out of the 700 images in the test set, NeuroXNet correctly classified 663 pictures, and the rest of the 37 were incorrectly classified. The few incorrect classifications could be caused by the model perceiving features of certain patient MRIs to be similar to multiple diseases making the model rely on a close probability value from the softmax function to diagnose the particular incorrect disease over the correct class. Otherwise, the model performed well, achieving an accuracy of $94.71 \%$ for multiclass diagnosis. 
medRxiv preprint doi: https://doi.org/10.1101/2021.12.13.21267728; this version posted December 14, 2021. The copyright holder for this preprint (which was not certified by peer review) is the author/funder, who has granted medRxiv a license to display the preprint in perpetuity.

It is made available under a CC-BY-NC 4.0 International license .

Other than the confusion matrix, another way to help see the model's overall performance on the test set is the classification report which gives the precision, recall, fl-score, and support for each of the classes with macro averaging as well as the weighted averaging. The classification report for the model is shown below:

\begin{tabular}{ccccc}
\hline \multicolumn{5}{c}{ Table 3: Classification Report for Diseases } \\
\hline & Precision & Recall & F1-score & Support \\
\hline AD & 0.92 & 0.92 & 0.92 & 100 \\
\hline $\mathrm{MCl}$ & 0.97 & 0.97 & 0.97 & 100 \\
\hline PD & 0.92 & 0.92 & 0.92 & 100 \\
\hline Glioma & 0.97 & 0.97 & 0.97 & 100 \\
\hline Meningioma & 0.96 & 0.96 & 0.96 & 100 \\
\hline Pituitary & 0.95 & 0.95 & 0.95 & 100 \\
\hline Normal & 0.94 & 0.94 & 0.94 & 100 \\
\hline
\end{tabular}

Table 3: Disease Classification Report

\begin{tabular}{|c|c|c|c|c|}
\hline & Precisio & ecall & F1-Score & Support \\
\hline Accuracy & & & 0.95 & 700 \\
\hline Macro Average & 0.95 & 0.95 & 0.95 & 700 \\
\hline Weighted Average & 0.95 & 0.95 & 0.95 & 700 \\
\hline
\end{tabular}

Table 4: Model Classification Report

Below are the formulas used in the Classification Report:

$$
\begin{gathered}
\text { Precision }=\frac{T P}{T P+F P}(\text { Equation } 1) \\
\text { Recall }=\frac{T P}{T P+F N}(\text { Equation } 2) \\
F 1=\frac{2 * \text { Precision } * \text { Recall }}{\text { Precision }+ \text { Recall }}(\text { Equation } 3) \\
\text { Accuracy }=\frac{T P+T N}{T P+F N+T N+F P}(\text { Equation } 4)
\end{gathered}
$$

The precision, recall, and $\mathrm{fl}$-score values for the model are $92 \%, 97 \%, 92 \%, 97 \%, 96 \%, 95 \%$, and $94 \%$ for AD, MCI, PD, Glioma, Meningioma, Pituitary, and Normal respectively. The accuracy, macro average, and weighted average of all the different methods of calculating performance are $95 \%$, with a support of 700 .

In addition, the Cohen's Kappa score for the model was calculated to be 0.9383 with the equation for the score shown below $\left(\mathrm{p}_{0}\right.$ represents relating observed agreement and $\mathrm{p}_{\mathrm{e}}$ represents the probability of chance agreement): 


$$
\text { Kappa Score }=1-\frac{1-p_{0}}{1-p_{e}}(\text { Equation } 5)
$$

The Matthews correlation coefficient was calculated to be 0.9383 with the equation for the coefficient calculation shown below:

$$
\text { Coefficent }=\frac{T P * T N-F P * F N}{\sqrt{(T P+F P)(T P+F N)(T N+F P)(T N+F N)}}(\text { Equation 6) }
$$

\subsection{NeuroXNet Treatment Analysis}

After NeuroXNet uses its CNN model to classify neurological diseases correctly, it uses nonimaging data inputted by the radiologist or primary physician to generate treatment recommendations. The algorithmic process that NeuroXNet uses in deciding which treatment to recommend was detailed in section 2. NeuroXNet had a $98 \%$ accuracy in predicting treatments plans with the highest survival rates.

In the final analysis, it was observed that the images of the training, validation, and testing sets all attained high accuracies and low loss values, signifying that the NeuroXNet has excellent potential in the diagnosis and treatment of neurological disorders.

\section{Conclusion}

This paper helped describe the layers and characteristics of the NeuroXNet model, which achieved a test accuracy of $94.71 \%$. This model is the first CNN model which approaches the diagnosis of neurodegenerative diseases, primarily Alzheimer's disease, Parkinson's disease, and Mild Cognitive Impairment and brain tumors (glioma, meningioma, and pituitary) through a novel deep learning architecture (NeuroXNet). The model helps find new blood biomarkers for the six diseases. Furthermore, NeuroXNet is the first to integrate a treatment component into the model and uses genomic data with the MRI images to diagnose patients. Through this model, doctors and radiologists can diagnose neurological diseases at an earlier stage and use the diagnosis in treating patients with the proper medications and treatment procedures, helping prevent the disease from progressing onto a deadlier stage that could affect the patient's health drastically. Consequently, this model has great potential to be used clinically and improve the lives of numerous patients. Through this paper, a new model is proposed and seen to attain a high accuracy that has many practical applications to radiology, neuroscience, and medicine, helping make a breakthrough in the diagnosis and treatment of neurological diseases using computational and biological approaches. 
medRxiv preprint doi: https://doi.org/10.1101/2021.12.13.21267728; this version posted December $14,2021$. The copyright holder for this preprint (which was not certified by peer review) is the author/funder, who has granted medRxiv a license to display the preprint in perpetuity.

It is made available under a CC-BY-NC 4.0 International license .

\section{References}

[1] D. Long et al., "Automatic classification of early Parkinson's disease with multi-modal mr imaging", PLoS ONE, vol. 7, no. 11, pp. e47714, 2012.

[2] Soltaninejad, S., Cheng, I., Basu, A.: Towards the identification of Parkinson's disease using only T1 MR images. In: Basu, A., Berretti, S. (eds.) ICSM 2018. LNCS, vol. 11010, pp. 145-156. Springer, Cham (2018).

[3] Chakraborty S, Aich S, Kim HC. Detection of Parkinson's Disease from 3T T1 Weighted MRI Scans Using 3D Convolutional Neural Network. Diagnostics (Basel). 2020 Jun 12;10(6):402. doi: 10.3390/diagnostics10060402. PMID: 32545609; PMCID: PMC7345307.

[4] F. E. Al-Khuzaie, O. Bayat, and A. D. Duru, "Diagnosis of Alzheimer disease using 2D MRI slices by convolutional neural network," Applied Bionics and Biomechanics, vol. 2021.

[5] Gorji, Hamed Taheri, and Naima Kaabouch. “A Deep Learning approach for Diagnosis of Mild Cognitive Impairment Based on MRI Images.” Brain sciences vol. 9,9 217. 28 Aug. 2019, doi:10.3390/brainsci9090217.

[6] Alzheimer's Association. (2021). 2021 ALZHEIMER'S DISEASE FACTS AND FIGURES. Alzheimer's Association.

[7] Statistics. Parkinson's Foundation. (n.d.). Retrieved September 15, 2021, from https://www.parkinson.org/Understanding-Parkinsons/Statistics.

[8] Mild cognitive impairment (MCI). Alzheimer's Disease and Dementia. (n.d.). Retrieved September 15, 2021, from https://www.alz.org/alzheimers-dementia/what-is-dementia/related_conditions/mildcognitive-impairment.

[9] Hanseeuw, BJ; Betensky RA, Jacobs HIL, Schultz AP, Sepulcre J, Becker JA, et al. Association of amyloid and tau with cognition in preclinical Alzheimer disease. JAMA Neurol 2019;76(8):915-24. 11.

[10] https://www.mayoclinic.org/diseases-conditions/parkinsons-disease/symptoms-causes/syc-20376055 
medRxiv preprint doi: https://doi.org/10.1101/2021.12.13.21267728; this version posted December $14,2021$. The copyright holder for this preprint (which was not certified by peer review) is the author/funder, who has granted medRxiv a license to display the preprint in perpetuity.

It is made available under a CC-BY-NC 4.0 International license .

[11] Folego G, Weiler M, Casseb RF, Pires R, Rocha A. Alzheimer's Disease Detection Through WholeBrain 3D-CNN MRI. Front Bioeng Biotechnol. 2020 Oct 30;8:534592. doi: 10.3389/fbioe.2020.534592. PMID: 33195111; PMCID: PMC7661929.

[12] Saha, Roshni, "Classification of Parkinson's Disease Using MRI Data and Deep Learning Convolution Neural Networks" (2019). Creative Components. 241.

[13] Ocasio E, Duong TQ. 2021. Deep learning prediction of mild cognitive impairment conversion to Alzheimer's disease at 3 years after diagnosis using longitudinal and whole-brain 3D MRI. PeerJ Computer Science 7:e560 https://doi.org/10.7717/peerj-cs.560

[14] Bhatele K. R, Bhadauria S. S. Classification of Neurodegenerative Diseases Based on VGG 19 Deep Transfer Learning Architecture: A Deep Learning Approach. Biosc.Biotech.Res.Comm. 2020;13(4)

[15] Tong T, Ledig C, Guerrero R, Schuh A, Koikkalainen J, Tolonen A, Rhodius H, Barkhof F, Tijms B, Lemstra AW, Soininen H, Remes AM, Waldemar G, Hasselbalch S, Mecocci P, Baroni M, Lötjönen J, Flier WV, Rueckert D. Five-class differential diagnostics of neurodegenerative diseases using random undersampling boosting. Neuroimage Clin. 2017 Jun 12;15:613-624. doi: 10.1016/j.nicl.2017.06.012. PMID: 28664032; PMCID: PMC5479966.

[16] Lin CH, Chiu SI, Chen TF, Jang JR, Chiu MJ. Classifications of Neurodegenerative Disorders Using a Multiplex Blood Biomarkers-Based Machine Learning Model. Int J Mol Sci. 2020 Sep 21;21(18):6914. doi: 10.3390/ijms21186914. PMID: 32967146; PMCID: PMC7555120.

[17] Gurpreet Singh, Meet Vadera, Lakshminarayanan Samavedham, and Erle Chuen-Hian Lim, Industrial \& Engineering Chemistry Research 201958 (26), 11498-11505.DOI:

10.1021/acs.iecr.8b06064

[18] Role of mris in detecting alzheimer's. Envision Radiology. (2020, July 21). Retrieved September 15, 2021, from https://www.envrad.com/role-of-mris-in-detecting-alzheimers/.

[19] Fioravanti, V., Benuzzi, F., Codeluppi, L., Contardi, S., Cavallieri, F., Nichelli, P., et al. (2015). MRI correlates of Parkinson's disease progression: a voxel based morphometry study. Parkinsons Dis. 2015:378032. doi: 10.1155/2015/378032 
[20] Velazquez, M.; Lee, Y.; Initiative, A.D.N. Random forest model for feature-based Alzheimer's disease conversion prediction from early mild cognitive impairment subjects. PLoS ONE 2021, 16, e0244773.

[21] Irmak, E. Multi-Classification of Brain Tumor MRI Images Using Deep Convolutional Neural Network with Fully Optimized Framework. Iran J Sci Technol Trans Electr Eng 45, 1015-1036 (2021). https://doi.org/10.1007/s40998-021-00426-9 W.-K.

[22] Sartaj Bhuvaji, Ankita Kadam, Prajakta Bhumkar, Sameer Dedge, and Swati Kanchan, "Brain Tumor Classification (MRI).” Kaggle, 2020, doi: 10.34740/KAGGLE/DSV/1183165.

[23] Friedman HS, Kerby T, Calvert H. Temozolomide and treatment of malignant glioma. Clin Cancer Res. 2000 Jul;6(7):2585-97. PMID: 10914698.

[24] Lee SY. Temozolomide resistance in glioblastoma multiforme. Genes Dis. 2016 May 11;3(3):198210. doi: 10.1016/j.gendis.2016.04.007. PMID: 30258889; PMCID: PMC6150109.

[25] Lamb, L.S., Pereboeva, L., Youngblood, S. et al. A combined treatment regimen of MGMTmodified $\gamma \delta$ T cells and temozolomide chemotherapy is effective against primary high grade gliomas. Sci Rep 11, 21133 (2021). https://doi.org/10.1038/s41598-021-00536-8

[26] “Brain Tumor Types, Glioblastoma, Meningioma \& More.” Miami Neuroscience Center, 21 Jan. 2020, https://miamineurosciencecenter.com/en/conditions/brain-tumors/types/.

[27] Silverberg, Gerald. "Brain Shunts Slow Alzheimer's Disease.” WebMD, WebMD, 22 Oct. 2002, https://www.webmd.com/alzheimers/news/20021022/brain-shunts-slow-alzheimers-disease.

[28] “Deep Brain Stimulation (DBS).” Parkinson's Foundation, https://www.parkinson.org/Understanding-Parkinsons/Treatment/Surgical-TreatmentOptions/Deep-Brain-Stimulation.

[29] “Duopa.” Parkinson's Foundation, https://www.parkinson.org/UnderstandingParkinsons/Treatment/Surgical-Treatment-Options/Duopa.

[30] H, Cameron M;Lonergan E;Lee. "Transcutaneous Electrical Nerve Stimulation (TENS) for Dementia." The Cochrane Database of Systematic Reviews, U.S. National Library of Medicine, https://pubmed.ncbi.nlm.nih.gov/12917999/. 
[31] Kieffer, Sara. "Meningioma Treatment: Johns Hopkins Meningioma Center.” Meningioma Treatment | Johns Hopkins Meningioma Center, 3 Dec. 2018, https://www.hopkinsmedicine.org/brain-tumor/specialty-centers/meningioma/treatment.html.

[32] "Surgery for Meningioma." Patient Care at NYU Langone Health, https://nyulangone.org/conditions/meningioma/treatments/surgery-for-meningioma.

[33] “Alzheimer's Journals: Dementia Journals: Parkinsonism: High Impact Articles: Scient Open Access." Alzheimer's Journals | Dementia Journals | Parkinsonism | High Impact Articles | Scient Open Access, https://scientonline.org/archive/23/journal-of-alzheimers-parkinsonism-anddementia.

[34] "Growth Factor Protects Key Brain Cells in Alzheimer's Models." UCSD News, https://ucsdnews.ucsd.edu/archive/thisweek/2009/02/16_GrowthFactorAlzheimers.asp.

[35] "Brain Shunts Slow Alzheimer's Disease.” WebMD, WebMD, 22 Oct. 2002, https://www.webmd.com/alzheimers/news/20021022/brain-shunts-slow-alzheimers-disease.

[36] "Levodopa and Carbidopa: Medlineplus Drug Information." MedlinePlus, U.S. National Library of Medicine, https://medlineplus.gov/druginfo/meds/a601068.html.

[37] Figge DA, Eskow Jaunarajs KL, Standaert DG. Dynamic DNA Methylation Regulates LevodopaInduced Dyskinesia. J Neurosci. 2016 Jun 15; 36(24):6514-24. doi: 10.1523/JNEUROSCI.068316.2016.PMID: 27307239; PMCID: PMC5015786.

[38] "Medications for Memory, Cognition and Dementia-Related Behaviors." Alzheimer's Disease and Dementia, https://www.alz.org/alzheimers-dementia/treatments/medications-for-memory.

[39] "Medication for Meningioma." Patient Care at NYU Langone Health, https://nyulangone.org/conditions/meningioma/treatments/medication-for-meningioma.

[40] Kieffer, Sara. "Medication (Drug Therapy): Treatment for Pituitary Tumors: Johns Hopkins Pituitary Tumor Center." Medication (Drug Therapy) | Treatment for Pituitary Tumors | Johns Hopkins Pituitary Tumor Center, 5 Oct. 2015, https://www.hopkinsmedicine.org/neurology_neurosurgery/centers_clinics/pituitary_center/pituitar y-tumor/treatment/drug-therapy.html. 
[41] "Stereotactic Radiosurgery as Effective in Eliminating Parkinson's Disease Tremors as Other Treatments but Less Invasive." ScienceDaily, ScienceDaily, 2 Nov. 2009, https://www.sciencedaily.com/releases/2009/11/091102121504.htm.

[42] Cuttler JM;Abdellah E;Goldberg Y;Al-Shamaa S;Symons SP;Black SE;Freedman M; "Low Doses of Ionizing Radiation as a Treatment for Alzheimer's Disease: A Pilot Study." Journal of Alzheimer's Disease : JAD, U.S. National Library of Medicine, https://pubmed.ncbi.nlm.nih.gov/33646146/.

[43] "Radiation Therapy for Pituitary Tumors." American Cancer Society, https://www.cancer.org/cancer/pituitary-tumors/treating/radiaton-therapy.html.

[44] "Meningioma." Mayo Clinic, Mayo Foundation for Medical Education and Research, 21 Apr. 2020, https://www.mayoclinic.org/diseases-conditions/meningioma/diagnosis-treatment/drc-20355648.

[45] "Radiation Therapy for Glioma." Memorial Sloan Kettering Cancer Center, https://www.mskcc.org/cancer-care/types/glioma/glioma-treatment/radiation-therapy-glioma.

[46] Schmidt M, Mock A, Jungk C, Sahm F et al. Transcriptomic analysis of aggressive meningiomas identifies PTTG1 and LEPR as prognostic biomarkers independent of WHO grade. Oncotarget 2016 Mar 22;7(12):14551-68. PMID: 26894859

[47] Dunn J, Lenis VP, Hilton DA, Warta R et al. Integration and Comparison of Transcriptomic and Proteomic Data for Meningioma. Cancers (Basel) 2020 Nov 5;12(11). PMID: 33167358

[48] Nilsson RJ, Balaj L, Hulleman E, van Rijn S et al. Blood platelets contain tumor-derived RNA biomarkers. Blood 2011 Sep 29;118(13):3680-3. PMID: 21832279

[49] Vierimaa O, Georgitsi M, Lehtonen R, Vahteristo P et al. Pituitary adenoma predisposition caused by germline mutations in the AIP gene. Science 2006 May 26;312(5777):1228-30. PMID: 16728643

[50] Sood S, Gallagher IJ, Lunnon K, Rullman E et al. A novel multi-tissue RNA diagnostic of healthy ageing relates to cognitive health status. Genome Biol 2015 Sep 7;16:185. PMID: 26343147

[51] Scherzer CR, Eklund AC, Morse LJ, Liao Z et al. Molecular markers of early Parkinson's disease based on gene expression in blood. Proc Natl Acad Sci U S A 2007 Jan 16;104(3):955-60. PMID: 17215369 
medRxiv preprint doi: https://doi.org/10.1101/2021.12.13.21267728; this version posted December 14, 2021. The copyright holder for this preprint (which was not certified by peer review) is the author/funder, who has granted medRxiv a license to display the preprint in perpetuity.

It is made available under a CC-BY-NC 4.0 International license .

[52] Scherzer CR, Grass JA, Liao Z, Pepivani I et al. GATA transcription factors directly regulate the Parkinson's disease-linked gene alpha-synuclein. Proc Natl Acad Sci U S A 2008 Aug 5;105(31):1090712. PMID: 18669654

[53] Maes OC, Xu S, Yu B, Chertkow HM et al. Transcriptional profiling of Alzheimer blood mononuclear cells by microarray. Neurobiol Aging 2007 Dec;28(12):1795-809. PMID: 16979800

[54] Maes OC, Schipper HM, Chertkow HM, Wang E. Methodology for discovery of Alzheimer's disease blood-based biomarkers. J Gerontol A Biol Sci Med Sci 2009 Jun;64(6):636-45. PMID: 19366883

[55] https://www.genecards.org/cgi-bin/carddisp.pl?gene=TOP2A

[56] Gao, Andrew. Identification of Blood-based Biomarkers for Early Stage Parkinson's Disease. 2020.10.22.20217893; doi: https://doi.org/10.1101/2020.10.22.20217893 